Revista de Filosofía

Volumen 66, (2010) 79 - 92

\title{
PIRRONISMO Y CONOCIMIENTO CIENTÍFICO
}

\author{
Armando Cíntora $G$. \\ Universidad Autónoma Metropolitana-Iztapalapa \\ cintora1@prodigy.net.mx
}

The difficulty is to realize the groundlessness of our believing.

LudWig WitTGENSTEIN

\begin{abstract}
Resumen
La búsqueda de justificación epistémica para los presupuestos metodológicos y axiológicos más básicos de la ciencia ha de enfrentarse al trilema de Agrippa. Se argumenta que la regresión al infinito -aun si esta regresión es solo potencial-y la circularidad no son las mejores alternativas del trilema y que el mejor disyunto es un dogmatismo minimalista, siendo uno de sus dogmas el siguiente condicional: Si nuestros presupuestos metodológicos y axiológicos más básicos (así como nuestros enunciados observacionales) de carácter cientifico fuesen los correctos, entonces las teorías científicas seleccionadas por estos presupuestos serían probable, o aproximadamente, verdaderas.
\end{abstract}

Palabras Clave: justificación epistémica, regresión al infinito, circularidad, dogmatismo, escepticismo pirrónico, relativismo.

\section{Abstract}

The search of epistemic justification for the most basic methodological and axiological presuppositions of science has to deal with Agrippa's trilemma.

It is argued that infinite regress - even if it is only a potential regress - and circularity are not the best alternatives of the trilemma and that the best disjunct is a minimalist dogmatism, with the following conditional, as one of its dogmas:

If our most basic methodological and axiological scientific presuppositions (as well as our observational statements) were the correct ones, then the scientific theories selected by these presuppositions would be the probably, or the approximately, true ones.

Keywords: epistémic justification, infinite regress, circularity, dogmatism, pyrrhonian scepticism, relativism. 


\section{Preámbulo}

L. Laudan afirmó durante su antiguo debate con J. Worrall ${ }^{1}$ :

... Lo que (...) reconforta al relativismo es un fracaso en lidiar con la pregunta: “¿cómo se justifican las reglas o estándares metodológicos?”

(Laudan 1989, pp. 369-70; énfasis mío).

R⿴囗十 Aquí vamos a argumentar que si el relativismo se sigue de la falta de justificación epistémica de nuestros presupuestos metodológicos más básicos, entonces el relativismo es imbatible, pues no parece que podamos decidir racionalmente cuáles cánones o criterios metodológicos últimos son objetivamente los correctos -como lo sugieren las dificultades perennes para justificar la inducción, la deducción, la abducción, nuestros fines epistémicos más básicos e inclusive la actitud racional misma.

Esto es, las dificultades para justificarlos sin caer en alguna de las inapetecibles alternativas del trilema pirrónico (es decir, el modo de regresión al infinito de Agrippa $\left.^{2}\right)$ : circularidad viciosa, regresión al infinito o dogmatismo, aunque todas estas alternatívas cuentan con paladines hoy en día.

Por ejemplo, algunos argumentan que la circularidad no siempre es viciosa ${ }^{3}$, en particular que no es viciosa la llamada circularidad de regla de inferencia-circularidad en la que una regla de inferencia se justifica a sí misma, directa o indirectamenteargumentan que este tipo de circularidad es el mejor disyunto entre aquellos de la trinidad pirrónica. Aquí se van a esbozar críticas a la regresión al infinito -aun si es solo una regresión potencial ${ }^{4}-\mathrm{y}$ a la circularidad (en tanto que tenga pretensiones justificatorias) y se argumentará en favor de un dogmatismo minimalista ${ }^{5} \mathrm{o}$, si se

Cf., los ensayos de L. Laudan (1989) y J. Worrall (1989) mencionados en la bibliografía. The Mode based upon regress ad infinitum is that whereby we assert that the thing adduced as a proof of the matter proposed needs a further proof, and this again another, and so on ad infinitum, so that the consequence is suspension [of assent], as we posses no starting-point for our argument ... We have the Mode based upon hypothesis when the Dogmatists, being forced to recede ad infinitum, take as their starting-point something which they do not establish but claim to assume as granted simply and without demonstration. The Mode of circular reasoning is the form used when the proof itself which ought to establish the matter of inquiry requires confirmation derived from the matter; in this case, being unable to assume either in order to establish the other, we suspend judgement about both. (Sextus Empiricus, pp. 166-69.)

3 Por ejemplo, D. Papineau (1992) defiende la circularidad de regla de inferencia como no viciosa, para una critica de la posición de Papineau, véase Cíntora (2005b.).

$4 \quad$ Una regresión potencial al infinito ha sido defendida recientemente por P. Klein (1999).

5 Un dogmatismo de fundamentos ya lo encontramos, por ejemplo, en Aristóteles (Post. An. $72 b 14$ y 72b18), así como en K. Popper (1945, p.218) y en J. Worrall (1989). 
prefiere, en favor de un austero axiomatismo convencionalista, entendiendo por dogma o axioma un presupuesto que se adopta como correcto o verdadero sin una justificación no viciosamente circular.

\section{Algunas respuestas internalistas al trilema de Agrippa}

Las justificaciones epistémicas argumentativas de corte internalista ${ }^{6}$ de nuestras reglas metodológicas más básicas y de nuestras proposiciones no evidentes (de existir proposiciones evidentes) han de lidiar con el trilema pirrónico. Esto es así, porque dado que todo argumento tiene premisas, la búsqueda de justificaciones argumentativas conduce a una regresión de más y más premisas por justificar. Y para detener la regresión, a la circularidad, o bien, al dogmatismo y la consecuente arbitrariedad lógica.

El dogmatismo (que consiste, por ejemplo, en creer, o al menos en adoptar, como verdadera una proposición, o en creer, o al menos en usar, como correcta una regla metodológica o de inferencia, sin justificación no circular) adolece de arbitrariedad lógica y es un tipo de fundacionismo en el que las proposiciones (y reglas metodológicas) básicas son creídas, o aceptadas, o adoptadas como verdaderas o correctas sin justificación epistémica alguna, es decir, dogmáticamente.

Por otro lado, la circularidad carece de eficacia justificatoria, tanto si se trata de una circularidad de premisa ${ }^{7}$ como de una circularidad de regla, ya que en una justificación argumentativa las premisas (el justificans) deben ser menos problemáticas, o mejor conocidas, que la conclusión: el justificando. La circularidad aparece en el tipo de coherentismo lineal, descrito por Aristóteles en sus Analíticos Posteriores ${ }^{8}$.

Existe un segundo tipo de coherentismo de carácter holista, que pretende que la justificación emerge, o sobreviene, conforme la red de razones deviene más extensa y apretada. Este coherentismo holista es, sin embargo, un caso de fundacionismo, un fundacionismo de un paso (como lo han discutido E. Sosa y P. Klein ${ }^{9}$ ), donde la propiedad fundacional es la pertenencia al conjunto coherente de razones. Este coherentismo holista, termina (como todo fundacionismo) en el dogmatismo y la

6 Para nosotros, una teoría internalista de la justificación epistémica será aquella que requiere que para que $\mathrm{S}$ tenga una creencia justificada $\mathrm{p}, \mathrm{S}$ apoye su creencia en $\mathrm{p}$ en los supuestos que la justifican; y que requiere que estos supuestos sean al menos en principio concientizables por el sujeto $\mathrm{S}$, es decir, que sean cognoscitivamente accesibles para $\mathrm{S}$. Lo que, por ejemplo, ocurriría si fuesen contenidos mentales de S. Cf., Fogelin (1994, pp. 119-121) y J. Dancy y Sosa (1994, pp. 132-133).

7 La circularidad de premisa es aquella en que la conclusión está contenida en alguna(s) de la(s) premisas.

$8 \quad$ Aristóteles, Analíticos Posteriores, I, iii, $73^{\mathrm{a}} 5$.

$9 \quad$ Cf., P. Klein 2005, p. 135. 
arbitrariedad lógica, una vez que exigimos una justificación para la relación entre la propiedad fundacional -en este caso, la pertenencia a un conjunto coherente de proposiciones- y la verdad.

Finalmente, la regresión al infinito, una alternativa que intenta eludir la arbitrariedad y la circularidad, parece ser una opción sicológicamente imposible para mentes finitas, aunque ya Karl Popper ${ }^{10}$ propuso un infinitismo potencial de 'tests' o críticas, para los enunciados observacionales ${ }^{11}$, en el primer tercio del siglo pasado.

En la propuesta de Popper, la regresión infinita se detiene cuando la comunidad científica, actuando como el jurado de un tribunal, conviene tentativamente que un enunciado observacional no es, por ahora, problemático, decidiendo que es inocente, ya que el enunciado observacional ha pasado tantas pruebas o críticas como esta comunidad considera pertinentes, juicio que a su vez dependerá del contexto pragmático. La comunidad científica conviene entonces aceptarlo de manera provisional como verdadero y de surgir nuevos argumentos críticos se podría continuar con la, en principio, interminable evaluación del enunciado observacional en cuestión.

Recientemente, Peter Klein también ha optado por una regresión al infinito potencial, pues Klein quiere eludir tanto la circuralidad viciosa del coherentismo aristotélico, así como la arbitrariedad, en tanto que pretendidamente verdaderas, de las proposiciones básicas de los diversos fundacionismos; y por lo tanto, Klein también rechaza el coherentismo de tipo holista, que es de hecho, un fundacionismo de un paso.

Según la propuesta infinitista de Klein, si surgiesen dudas sobre el valor de verdad de una proposición no evidente $p$ y que por ende quisiésemos justificarla, procederíamos ofreciendo una secuencia no repetitiva y no cerrada en sí misma -es decir, potencialmente infinita- de diferentes buenas razones que apoyasen a $p$. Y cada razón de esta sucesión sería una razón en favor de la razón que le sigue en la cadena, aun cuando estas razones pretendidamente justificadoras, carezcan de justificación, antes

11 Popper (1977) rechazó el psicologismo, la tesis de que las experiencias preceptuales pueden justificar los enunciados observacionales. Para Popper las experiencias perceptivas solo pueden motivar o causar los enunciados observacionales, pero no justificarlos. Y Popper argumentó que los enunciados observacionales solo pueden ser justificados infiriéndolos de otros enunciados. Esta postura argumentativa en cuanto a la justificación epistémica ineludiblemente conduce al trilema pirrónico, de ahí que para detener la regresión, al menos por un tiempo, Popper haya propuesto las decisiones convencionales de la comunidad científica, decisiones que declararían como tentativamente no problemáticos, en cuanto a su verdad, a ciertos enunciados observacionales. Estas decisiones, aunque convencionales, no serían arbitrarias, pues los enunciados observacionales elegidos como no problemáticos deben ser fácilmente contrastables a la luz del conocimiento científico entonces aceptado y deben haber sobrevivido a la crítica intersubjetiva. Entonces, estos enunciados singulares aceptados convencionalmente como verdaderos tienen el carácter de dogmas provisionales. 
de su ingreso a la cadena de razones. Por otro lado, la relación de las diversas razones entre si y con $p$ no es necesariamente de carácter inferencial, sino que también puede ser, por ejemplo, explicativa.

Klein afirma que la justificación de $p$ se acrecienta conforme crece la cadena de razones no repetitivas que apoyan a $p$; no habiendo una mera transmisión inferencial, o herencia, de la justificación, pues las razones ofrecidas a favor de 'p' carecen de justificación, antes de ingresar a la cadena. Así, esta propuesta es similar al coherentismo de justificación emergente, salvo que para Klein el diámetro argumental es potencialmente infinito.

Por otro lado, se exige que el infinito potencial de razones esté subjetivamente 'disponible,' es decir, debe existir la disposición o capacidad para creer en, o para sostener, las diversas e innumerables razones en favor de $p$; y estas razones deben serlo para nosotros en un tiempo $t$, aunque nunca lleguemos a considerar conscientemente la mayoría de estas razones: aunque solo algunas de estas razones, subjetivamente disponibles, sean de hecho aducidas a favor de la proposición $p$. Así mismo, las razones aducidas a favor de $p$ no han de estar socavadas por razones también subjetivamente disponibles.

Este infinito potencial de razones, se dice, no proporciona una justificación plena de $p$, pero sí hace más razonable el creer, que el no creer, que $p$, evitándose así una suspensión pirrónica del juicio o epojé en cuanto a la verdad de $p$, precisamente porque supuestamente la justificación se incrementa, va emergiendo, conforme crece la secuencia de razones no previamente justificadas que apoyan a $p$.

\section{II.1. Dificultades de la posición infinitista propuesta por P. Klein}

Menciono tres criticas, que podrían ser originales, a esta posición, varias otras criticas están reseñadas en la literatura pertinente ${ }^{12}$ :

i) De haber, de surgir, justificación, cómo explica y cómo justifica el infinitismo la emergencia de la justificación, es decir, ¿cómo y por qué la justificación sobreviene y se incrementa conforme la cadena de razones pretendidamente justificatorias se alarga?

Klein simplemente afirma que la justificación emerge y se incrementa conforme aumentamos las razones, aun cuando estas razones no han sido a su vez previamente justificadas, pero Klein no justifica esta afirmación, de modo que su afirmación tiene un carácter dogmático. Y esto sería así, aun si Klein argumentase que simplemente está describiendo nuestra práctica epistémica, pues descripción no es justificación.

La justificación epistémica tiene un carácter normativo que no parece posible obtener de una pura descripción, pues no hay descripciones puras; así, al seleccionar 
algunas prácticas epistémicas como dignas de descripción tácitamente hacemos un juicio de valor en su favor, juicio de valor que a su vez requiere justificación.

Si Klein ha de evitar la arbitrariedad, debe justificar como confiables lo que el da por sentado como nuestros usos epistémicos, en tanto fuentes y transmisores de verdades y para esta justificación no basta la mera descripción de nuestros hábitos y costumbres epistémicos, aun si esta descripción fuese una descripción correcta de nuestras prácticas, pues aun así habría que justificar nuestra práctica como objetivamente confiable. Brevemente, aun si todos nosotros tuviésemos una propensión a formar creencias de cierta forma, este hecho por sí mismo no mostraría que debamos formar nuestra creencias de esta manera.

La pregunta de cómo y por qué emerge la justificación es pertinente, dado que para este infinitismo no se trata solo de transmitir inferencialmente la justificación desde algunas últimas razones básicas. Recuérdese que Klein ha rechazado el fundacionismo, porque cree que las pretensiones de verdad de las proposiciones básicas del fundacionismo son lógicamente arbitrarias ${ }^{13}$, ya que no se justifican, en tanto que verdaderas.

Pero ahora hemos descubierto un problema análogo en el infinitismo de Klein, pues no se justifica la supuesta emergencia e incremento de justificación conforme crece la cadena de razones, previamente carentes de justificación. Simplemente se da por sentada esta emergencia, lo que introduce la arbitrariedad lógica, algo que Klein parecía querer eludir, dado que esta fue una de sus críticas al fundacionismo.

ii) Si hemos de evitar la arbitrariedad, las razones aducidas a favor de 'p' deben de ser buenas razones, y para justificarlas, para evaluarlas como buenas razones requeriremos criterios, presupuestos metodológicos, que también han de ser justificados ${ }^{14}$.

Sobre cómo justificar estos criterios Klein tiene muy poco que decir, salvo que da la bienvenida a la circularidad criteriológica. Una circularidad, en la que los diversos métodos o criterios de evaluación son usados, directa o indirectamente, en su the base, that is, a reason for which there are no further reasons making it even slightly better to accept than any of its contraries (P. Klein 1999, p. 297.)

14 El enfoque aquí seguido es 'metodista', es decir, suponemos que requerimos criterios o métodos racionales, si hemos de poder justificar como buenas razones, nuestras razones a favor de $p$. La alternativa particularista, que presupone que podemos identificar como justificadas, o inclusive como conocidas, algunas razones, aun en ausencia de, sin aplicar, criterios o métodos racionales, hace una petición de principio en contra del escéptico. Así,

The particularist thus tends to be antiskeptical on principle. But the methodist is as such equally receptive to skepticism and to the contrary.

(Sosa 1991, p. 166.) 
propia justificación ${ }^{15}$. Klein cree, como otros muchos (por ejemplo, David Papineau en el caso de la inducción y Paul Boghossian para el modus ponens), que la circularidad criteriológica, que la circularidad de regla metodológica, no es viciosa; Klein cree que la consistencia entre nuestras prácticas epistémicas y nuestras creencias exige la circularidad metodológica y que por lo tanto esta circularidad no es viciosa, creencia esta última que es, o al menos parece ser, una estipulación ad hoc. Amén de que se presupone que nuestras prácticas epistémicas son objetivamente confiables.

La circularidad criteriológica crea, sin embargo, la posibilidad de un relativismo criteriológico en el que, por ejemplo, el 'afirmar el consecuente' y la contrainducción podrían ser correctas, pues estas reglas de inferencia también pueden ser justificadas circularmente ${ }^{16}$.

iii) Finalmente, esta posición requiere convenciones de carácter pragmático para decidir cuando la regresión de buenas razones ha proporcionado, por ahora, una justificación suficientemente buena. Y si estas convenciones no han de ser arbitrarias, o solo aceptadas dogmáticamente, también deben ser justificadas.

Pero la justificación de estas convenciones de primer orden requerirá, a su vez, de una cadena de razones potencialmente infinita y para decidir dónde detener esta segunda cadena, se requerirán convenciones de segundo orden, generándose así una regresión de convenciones y un sinnúmero de cadenas supuestamente justificatorias de orden superior, todas ellas con longitudes potencialmente infinitas.

De modo que la justificación de nuestra proposición no evidente $p$ requerirá una infinidad de diferentes secuencias supuestamente justificatorias y con cada una de estas secuencias -razones subjetivamente disponibles- con una longitud potencialmente infinita. Ésta es una tarea que es, o que parece ser, sicológicamente imposible, excepto si adoptásemos las convenciones sin justificación alguna, es decir, excepto si las aceptásemos dogmáticamente.

15 ... Any sufficiently careful and self-conscious person who believes that his/her use of Mtype belief acquisition methods are reliable will employ those very methods in acquiring the belief in the conclusion. But that is not a fallacy of circular reasoning. It is merely making one's practices consistent with one's beliefs. If I thought that my reliable belief acquisition methods were M-type methods, it would be imprudent of me, to say the least, to use some other method of acquiring beliefs - no matter what the beliefs were. Indeed, I would legitimately be accused of an inconsistency between my practices and my beliefs. (P. Klein 2004, p. 115) (Se agregó énfasis).

16 Cf., Cíntora 2005b, cap. I . 


\section{Otras respuestas al trilema de Agrippa}

Hasta aquí hemos repasado superficialmente las teorías de la justificación epistémica de corte internalista, pero las justificaciones externalistas de corte confiabilista ${ }^{17}$ también tienen que enfrentar el trilema pirrónico, una vez que se requiere una justificación de la supuesta confiabilidad de los diversos procesos o facultades, fuentes y transmisores de verdades. Si buscamos una justificación de estos procesos y facultades cognoscitivos caemos de nuevo en el trilema ${ }^{18}$. De ahí que Sosa parezca proponer validar todo conocimiento solo una vez que se dan por sentados como en general confiables la intuición racional, la memoria, la percepción, la introspección, así como la deducción, la inducción y la abducción ${ }^{19}$.

Por otro lado, y repitiendo una crítica frecuente, las justificaciones externalistas no parecen proporcionar una adecuada explicación del mejor conocimiento humano adulto, ya que no son autorreflexivas, es decir, ya que no parecen ser epistémicamente responsables.

17 Una justificación es externalista si permite que al menos algunos de los factores justificadores de una proposición no sean cognitivamente accesibles para el sujeto cognoscente, de modo que puedan ser externos a la perspectiva cognoscitiva del sujeto. Y un externalismo de corte confiabilista exige además que la creencia justificada sea producida por un proceso que haga objetivamente probable que ésta sea verdadera, pero sin requerir que el sujeto que tiene la creencia justificada tenga acceso cognoscitivo a la relación de confiabilidad en cuestión. Esto último es lo que le da a la postura confiabilista un carácter externalista (cf., J. Dancy \& E. Sosa 1994, pp. 132-6.)

18 Por ejemplo, E. Sosa comenta:

(...) ¿cómo podría justificarse la adición de una facultad a la lista [a la lista de facultades putativamente confiables] sino es mediante el uso -directo o indirecto- precisamente de esa facultad? ¿Y no es eso tan viciosamente circular como el declarar a una fuente como confiable aceptando sin más sus reportes e infiriendo que entrega verdades? Tal razonamiento no es confiable y en todo caso es inaceptable. Quizá podríamos evitar la circularidad viciosa permitiendo que una facultad obtenga apoyo a partir del uso de otras facultades. Pero estas necesitarían su propio apoyo y ¿cómo podrían obtenerlo sino mediante el apoyo de unas en otras? Así pues el confiabilismo es llevado a buscar refugio en un circulo suficientemente amplio, que debe considerar como benigno, quizá debido a su diámetro amplio (Sosa 1991, p. 95; énfasís mío).

19 Sosa nos dice:

Radical rationalism admits only (racional) intuition and deduction (along with memory) as its faculties of choice (or anyhow of top choice) and wishes to validate all knowledge in terms of these faculties; thus the Cartesian grand project. Virtue perspectivism admits also perception and introspection along with intuition and deduction, as well as inductive and abductive reasoning (Sosa 1997, p. 423). 


\section{III.1. Un dogmatismo minimalista}

Ante las dificultades del coherentismo, el infinitismo y las posturas externalistas parece que un mínimo de presupuestos dogmáticos o axiomas ${ }^{20}$-en particular un dogmatismo respecto a algunos de los presupuestos metodológicos más básicos de la ciencia- podría ser malgré tout la mejor alternativa. Así, por ejemplo,

No veo otra salida que la afirmación dogmática de que conocemos el principio inductivo u otro equivalente; la otra alternativa es tirar todo lo que la ciencia y el sentido común consideran como conocimiento

(B. Russell 1943, p. 683.)(Se agregó el énfasis).

Este tipo de dogmatismo minimalista es intelectualmente honesto, pues concede con toda franqueza que ha de recurrir a presupuestos no justificados, porque estos presupuestos parecen ser injustificables (i.e., sin circularidad o regresión viciosas y sin recurrir a intuiciones u otros supuestos no justificados), como correctos o verdaderos. Este dogmatismo minimalista de algunas proposiciones básicas y de algunos presupuestos metodológicos últimos se sigue (si omitimos el infinitismo como una alternativa apropiada) de los siguientes tres principios:

1. En las justificaciones de enunciados o de reglas de inferencia evitese la circularidad $^{21}$ : tanto la circularidad de premisa ${ }^{22}$, como la circularidad de reglas de inferencia.

2. En las justificaciones evitese la arbitrariedad lógica. Toda razón justificadora de un enunciado o creencia requiere a su vez de otra razón, salvo en el caso de los mínimos presupuestos últimos. Estos presupuestos últimos podrán ser aceptados sin razones ulteriores.

3. De entre los dogmatismos prefiérase uno minimalista, en particular, óptese solo por los dogmas necesarios y suficientes para la empresa científica y no más ${ }^{23}$.

20 O bien, podríamos considerarlos como convenciones, aceptadas como correctas o verdaderas, como resultado de una decisión, de una estipulación.

21 El dogmatismo y la circularidad son lógicamente equivalentes -aunque pueden diferenciarse en el ámbito sicológico- ya que solo difieren en el tamaño del diámetro del circulo argumentativo, siendo este diámetro argumentativo mínimo en el caso del dogmatismo ( $p$ porque $p$, una reiteración) y en general más amplio en el caso de la circularidad. Y mientras que el dogmatismo concede abiertamente que requiere dogmas -es decir, de presupuestos no justificados- aquellos que echan mano de la circularidad frecuentemente lo hacen tácitamente; de ser así, los paladines de la circularidad son dogmáticos de closet.

22 Otra vez, la circularidad de premisa es aquella en que la conclusión está contenida en alguna de las premisas, en un sentido apropiado de contención.

23 Es decir, óptese por los dogmas metodológicos necesarios y suficientes para confirmar, corroborar, refutar o, en general, evaluar, las teorías científicas como verdaderas, o probablemente verdaderas, o si se prefiere, como empíricamente adecuadas. 
(Cuáles pueden ser los necesarios y suficientes es un debate abierto, como lo ilustran la pluralidad de caracterizaciones de la metodología científica, por ejemplo, ahí están los disensos metodológicos entre realistas científicos y constructivistas empíricos). Esta es una prescripción en favor de la economía de presupuestos dogmáticos ${ }^{24}$.

De estos principios se sigue que un mínimo de presupuestos últimos han de ser aceptados sin justificación, es decir, como dogmas o axiomas. Aristóteles ya tenía esto claro, por ejemplo, cuando afirmó que no se podía demostrar la ley de la no contradicción ${ }^{25}$ y Wittgenstein cuando afirmó,

At the foundation of well-founded belief lies belief that is not founded.

(Wittgenstein, On Certainty, 253).

Y

... The questions that we raise and our doubts depend on the fact that some propositions are exempt from doubt, are as it were like hinges on which those turn.

(Wittgenstein, On Certainty, 341).

Esto, muy a pesar de Laudan y su intento de combatir un relativismo de fundamentos resultado de la falta de justificación de estos fundamentos, aunque de hecho, la meta-metodología de Laudan también presupone sin justificación una regla inductiva por enumeración (a la que él llama R1), la deducción, algunos cánones "pre-filosóficos" de éxito científico, de carácter pragmático, así como, un esquema para la racionalidad de medios y fines ${ }^{26}$. Entonces, si caracterizamos al relativismo como lo hace Laudan, el relativismo gana.

El dogmatismo minimalista crea, empero, la posibilidad de un relativismo de diferentes dogmas, axiomas o convenciones, lógica, y quizá también sicológicamente,

24 En su Open Society (1945, cap. XXIV), Popper ofreció un argumento moral a favor de un dogmatismo minimalista (a favor de lo que él consideraba un irracionalismo minimalista).

25 Nosotros acabamos de reconocer que es imposible ser y no ser al mismo tiempo, y fundados en esta imposibilidad hemos declarado que nuestro principio es el principio cierto por excelencia.También hay filósofos que, dando una muestra de ignorancia, quieren demostrar este principio; porque es ignorancia no saber distinguir lo que tiene necesidad de demostración de lo que no la tiene157. Es absolutamente imposible demostrarlo todo, porque sería preciso caminar hasta el infinito; de suerte que no resultaría demostración. Y si hay verdades que no deben demostrarse, dígasenos qué principio, como no sea el expuesto, se encuentra en semejante caso.

(Aristóteles 1999, 4.4. Se agregó énfasis).

26 Cf., Cíntora 2005a, caps. V y VI. 
arbitrarios, pues nuestras intuiciones metodológicas distan de ser universales, como lo muestra el caso de los creacionistas "científicos", quienes difieren de nosotros en algunos presupuestos metodológicos muy básicos o, al menos, divergen de nosotros en su jerarquización de posibles presupuestos metodológicos compartidos. Así, algunos creacionistas "científicos" priorizan, anteponen, la coherencia entre nuestra teorías científicas y una lectura literal del Génesis sobre la prescripción metodológica de preferir hipótesis auxiliares no ad hoc acrecentadoras de poder predictivo. Estos creacionistas también sobreponen la consistencia con las Escrituras a las prescripciones pragmáticas que favorecen, en igualdad de otras virtudes epistémicas, las teorías más sencillas y elegantes -aunque sencillez y elegancia teórica son conceptos oscuros y muy debatidos.

Si se nos preguntase si nuestros dogmas metodológicos últimos son verdaderos o correctos, no parece que podamos contestar afirmativamente sin una petición de principio. Sobre su corrección objetiva parece que habrá que suspender el juicio ${ }^{27}$, lo que no obsta para que los usemos, dado que forman parte de nuestra tradición, de nuestros usos y costumbres epistémicos. Los usamos sin creerlos, los adoptamos pasivamente, los aceptamos sin entusiasmo (análogamente a quienes siguen prácticas rituales, religiosas o cívicas, sin creer, ni rechazar, incluso ignorando, las creencias detrás de sus prácticas rituales. Se siguen, se aceptan estas prácticas con indiferencia o ignorancia epistémica). Los usamos conscientes de que aunque nuestros usos y costumbres epistémicos pueden inducirnos a adoptar o creer en nuestros métodos, esta aceptación o creencia no los justifica como verdaderos o correctos. De lo que se sigue que no somos plenamente responsables en el ámbito epistémico, porque parece que hay límites lógicos (el trilema de Agripa) a qué tanta responsabilidad epistémica podemos alcanzar.

27 Dado nuestro fracaso hasta ahora para justificar estos presupuestos básicos y considerando el trilema pirrónico, las posibilidades de encontrar esta justificación en el futuro no son promisorias, aunque para llegar a esta conclusión pesimista hemos argumentado usando las mismísimas facultades y métodos cognoscitivos de cuya confiabilidad dudamos, entonces los argumento escépticos serán tan dudosos como dudosa sea la confiabilidad de nuestra capacidades cognoscitivas. Esto ya lo tenía claro Sexto con sus analogías: i) entre la función de los argumentos escépticos y la función de una purga, la purga se elimina a sí misma junto con otros detritos, análogamente los argumentos escépticos se eliminan a sí mismos junto con las afirmaciones dogmáticas de conocimiento que han socavado; y ii) su analogía entre los argumentos escépticos y una escalera, escalera que una vez usada puede ser desechada. Después de la purga, o de haber tirado la escalera, lo que queda es la epojé pirrónica, no solo respecto a la putativa corrección de las afirmaciones del dogmático, sino también respecto de la corrección de los argumentos mismos del escéptico. Así, aun si el pirronismo fuese correcto, no podría ser justificado como correcto. 
Esta fue, o parece que fue, la actitud pirrónica, de $\operatorname{Sexto}^{28}$, de Montaigne $^{29}$ et al. Nótese, por otro lado, que las dudas sobre la justificabilidad de nuestros presupuestos metodológicos infectarán mucho de lo que damos por sentado como conocimiento científico, dado el carácter tan básico de estas reglas metodológicas.

De modo que parece que no sabemos, porque no parece que podamos justificar y por lo tanto saber ${ }^{30}$, si estos presupuestos metodológicos últimos son los correctos, pero los usamos con la esperanza de que sean los correctos, lo que por cierto, bien puede ser. Esta es una esperanza a-racional, pues es una esperanza que tampoco parece que podamos justificar sin circularidad o regresión viciosas.

De ser así, habría que reformular las teorías y resultados respaldados por la investigación científica como condicionales de la forma: ' $s i$ nuestros presupuestos metodológicos, reglas de inferencia y principios epistémicos más básicos (así como nuestros enunciados observacionales) fuesen justificados como en general los correctos (lo que bien pudiesen ser), entonces las teorías, las conclusiones, confirmadas o corroboradas por nuestras investigaciones científicas, podrían ser justificadamente creídas como las mejores candidatas a ser verdaderas, o como las más aproximadas a la verdad, o si se prefiere, como las más adecuadas empíricamente' ${ }^{31}$. Claro que esta afirmación presupone que el condicional es correcto, algo que tampoco podemos justificar sin enfrentarnos al trilema, de modo que este condicional podría ser uno de los dogmas más básicos requeridos para hacer ciencia ${ }^{32}$.

Pero, por otro lado, los crecientes éxitos científico-tecnológicos de los últimos siglos minan las dudas escépticas y la rueda argumentativa da otra vuelta: y el peñasco de Sísifo vuelve a rodar cuesta abajo. Y terminamos con un impasse.

Por cierto, estas dificultades de la racionalidad teórica para encontrar justificaciones de nuestros presupuestos metodológicos y epistémicos más básicos, también

28 Por ejemplo, Frede nos dice: ... "Since the skeptic wants to see whether his opponent at least by his own standards or canons has knowledge, he in his own arguments adheres to these standards. But this does not mean that he himself is committed to them" (Frede 1987, p. 204).

29 Cf., R. H. Popkin 1979, cap. III.

30 De requerir el conocimiento de la justificación, como suele afirmarse.

31 Este condicional no es trivialmente verdadero, pues aun si el antecedente fuese verdadero, el consecuente podría ser falso, por ejemplo, cuando los elementos en el antecedente no fuesen suficientes para lograr la justificación de las teorías científicas o cuando no fuesen aplicados correctamente.

32 Nótese que los dogmas metodológicos más básicos de la ciencia (tales como nuestro condicional, la deducción, la inducción, la abducción, etc.) tampoco parece que puedan ser criticados o puestos en duda sin presuponerlos, de modo que parecen ser tanto no justificables, como no criticables. 
las encontramos en el ámbito de la racionalidad práctica, al buscar justificaciones -no viciosamente circulares- de nuestras metas últimas como deseables ${ }^{33}$.

\section{Referencias bibliográficas}

Aristóteles (1999), Metafisica (traducción de Patricio de Azcárate) Alicante: Biblioteca Virtual Miguel de Cervantes. (http://www.cervantesvirtual.com/ servlet/SirveObras/12260620880115953087846/index.htm). N. sobre edición original: Edición digital basada en la edición de Miguel Candel, Madrid, Espasa Calpe, 1997.

Boghossian, P. (2001), "How are objective epistemic reasons possible?” Philosophical Studies. 106: 1-40.

Cíntora, A. (2005a), Los presupuestos irracionales de la racionalidad. Barcelona: Anthropos.

(2005b), “Es legitima la justificación regla-circular de la inducción?” Signos Filosóficos. XIII: 57-71. México.

Dancy, J. y Sosa, E. (1994), A Companion to Epistemology. Oxford/Cambridge MA.: Blackwell.

Frede, M. (1987), “The Skeptic's Beliefs", en Essays in Ancient Philosophy. Minneapolis: University of Minnesota Press; pp. 179-200.

Fogelin, J. R. (1994), Pyrrhonian Reflections on Knowledge and Justification. New York, NY: Oxford University Press.

Klein, P. D. (1999), "Human Knowledge and the infinite regress of reasons", en J. Tomberlin, ed., Philosophical Perspectives. 13. Malden, MA/Oxford: Blackwell.

(2004), "Skepticism: Ascent and Assent?" en Ernest Sosa and his Critics, John Greco, ed., Oxford: Blackwell.

(2005), "Is infinitism the solution to the regress problem?", en Contemporary Debates in Epistemology, M. Steup y Ernest Sosa, eds., Malden, MA/ Oxford: Blackwell.

33 Cf., Cíntora (2005a), capítulo VI. Así mismo, diversos estudios psicológicos concluyen que al tomar decisiones los individuos frecuentemente parecen proceder de manera aracional, así,

During the 1970s ... It became increasingly apparent that people do not typically solve problems, make decisions, or reach conclusions using the kinds of standard, conscious, and rational processes that they were more-or-less assumed to be using (...) When people were observed making choices and solving problems of interesting complexity, the rational and logical elements were often missing (A. S. Reber 1993, p. 13). 
Laudan, L. (1989), "If it ain't broke, don't fix it". British Journal for the Philosophy of Science. 40: 369-375.

Papineau, D. (1992), "Reliabilism, Induction and Scepticism". The Philosophical Quarterly. 42: 1-20.

Popkin, R. H. (1979), The History of Scepticism: from Erasmus to Spinoza. Berkeley/ Los Angeles: University of California Press.

Popper, K. (1945), The Open Society and its Enemies. Londres: Routledge.

(1977), La Lógica de la Investigación Cientifica. Madrid: Tecnos.

Reber, A. S. (1993), Implicit Learning and Tacit Knowledge: An Essay on the Cognitive Unconscious. New York, New York: Oxford University Press.

Russell, B. (1943), "Reply to Critics", en P. A. Schilpp, ed., The Philosophy of Bertrand Russell. La Salle: Open Court.

Sextus Empiricus, (1967), Outlines of Phyrronism, traducido por R. G. Bury, Cambridge, Mass.: Harvard University Press.

Sosa, E. (1991a), "Nature Unmirrored, Epistemology Naturalized" en Knowledge in Perspective. Selected Essays in Epistemology. Cambridge/New York, New York: Cambridge University Press.

(1991b), "The Raft and the Pyramid" en Knowledge in Perspective. Selected Essays in Epistemology. Cambridge/New York, New York: Cambridge University Press.

(1997), "Reflective Knowledge in the Best of Circles". The Journal of Philosophy. 94: 410-430.

Wittgenstein, L. (1969), On Certainty. Oxford: Basil Blackwell.

Worrall, J. (1989), "Fix it and be damned: a reply to Laudan". British Journal for the Philosophy of Science. 40: 376-388. 